\title{
Formation and Structure of Organized Molecular Films of Organo-modified Montmorillonite
}

\author{
Jun-ichi Kusaka ${ }^{1}$, Rintaro Nomura ${ }^{2}$, and Atsuhiro Fujimori ${ }^{2, *}$ \\ ${ }^{1}$ Department of Polymer Science and Engineering, and ${ }^{2}$ Graduate School of Science and Engineering, Yamagata University, \\ Yonazawa, Yamagata, 992-8510, Japan \\ Fax: +81-238-26-3073, e-mail: fujimori@yz.yamagata-u.ac.jp
}

Molecular arrangement and morphology of Langmuir-Blodgett (LB) films for the several organo-modified montmorillonites were investigated by out-of plane and in-plane X-ray diffraction (XRD), and atomic force microscopy (AFM). These organo-modified montmorillonites are formed by cation exchange reaction with quaternary ammonium regents containing octadecyl chain. The organo-modified montmorillonites formed extremely condensed monolayer on the water surface estimated by surface pressure-area $(\pi-A)$ isotherms. From the results of out-of plane XRD, formation of highly ordered layer structure was confirmed in these clay LB film. Further, two-dimensional lattice of long alkyl chain of organo-modified parts packed hexagonally or orthorhombically in the films. Surface morphology of Z-type monolayers on solid of organo-modified montmorillonites indicates heterogeneous modification ratio in montmorillonite surface by tapping mode AFM observation.

Key words: Langmuir-Blodgett film, organo-modified montmorillonite, molecular arrangement, surface morphology

\section{INTRODUCTION}

Clay minerals have played important roles in the modern technology. For example, new materials of functional ceramics are made from mica. ${ }^{1} \quad$ Organo-clay composites have been extensively investigated in material science. ${ }^{2}$ Thin films of clay minerals have been studied in application to modified electrodes, sensors, photochromic devices, nonlinear optical devices, and so on. ${ }^{3}$ The Langmuir-Blodgett (LB) method is one of the most useful techniques to prepare ultra-thin films. ${ }^{4}$ In this method, floating molecular monolayers formed at an air/water interface are deposited on a solid substrate in a layer-by-layer way. Ultra-thin films of clay minerals prepared by the LB method have been reported. ${ }^{5}$ Recently, the clay LB films have been hybridized with alkylammonium cations. ${ }^{6} \quad$ Smectite clay is exfoliated into single clay sheets with the negative charge in an aqueous suspension at a low concentration. ${ }^{7}$ When a solution of an amphiphilic alkylammonium salt is spread onto the clay suspension, the negatively charged clay platelets in the suspension are adsorbed onto a floating monolayer of the alkylammonium cation at an air-clay suspension interface. The hybrid monolayers of the clay platelets and the ammonium cations formed at the interface can be transferred onto a solid surface to fabricate a hybrid multilayer. Interestingly, a floating monolayer of a water-soluble alkylammonium cation is stabilized at the air-suspension interface by the hybridization with the clay platelets. ${ }^{6}$ The surface pressure-area $(\pi-A)$ isotherm ofthe hybrid monolayer is dependent on the clay concentration in the suspension: as the concentration is higher, the isotherm shifts to the larger area side. ${ }^{6}$ This suggests that structure and properties of the hybrid mono- and (a)

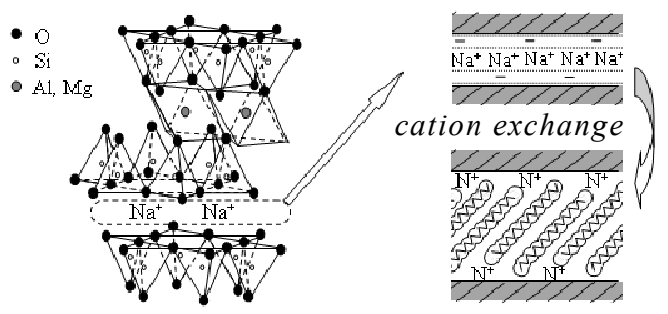

(b)<smiles>CCCCC(C)(C)Cc1ccccc1</smiles>

(C)<smiles></smiles>

Figure 1 (a) Illustration of structure of montorillonite before and after cation exchange reaction. (b) Chemical structure of quaternary ammonium regents; (A) dimethyl methylbenzile octadecyl (DMPO), (B) dimethyl dioctadecyl (DMDO), (C) methyl hydroxy ethyl octadecyl (MHEO), and (D) trimethyl octadecyl (TMO) ammonium cations. 
(a)

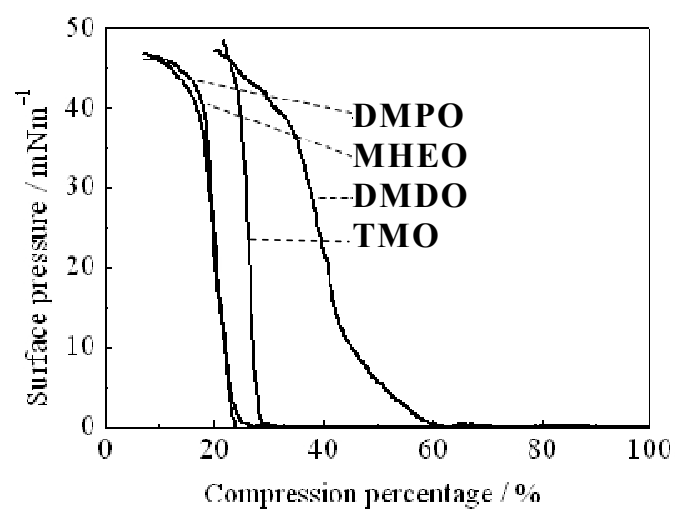

(b)

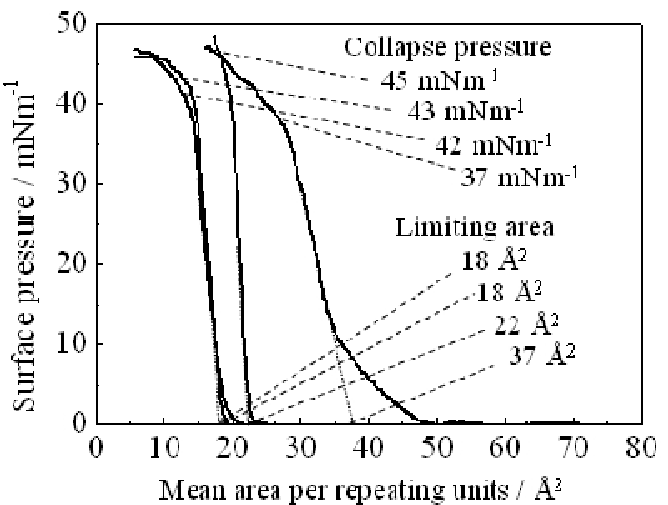

Figure $2 \pi$-A isotherms of monolayers for organo-modified clays on the water surface $\left(15{ }^{\circ} \mathrm{C}\right) ;$ (a) compression percentage $v s$. surface pressure (b) mean area per repeating units $v s$. surface pressure.

multi-layers would be varied depending on the clay concentration in the suspension. In this work, floating molecular monolayers and ultra-thin films on solid of pre-formed morgano-modified montmorillonites (MMTs) were constructed by LB method. These LB multilayers of organo-modified MMTs were characterized by the out-of plane and in-plane $\mathrm{X}$-ray diffraction (XRD) methods and atomic force microscopic (AFM) observation.

\section{EXPERIMENTAL}

\subsection{Materials}

Natural $\mathrm{Na}^{+}$-montmorillonite was kindly supplied by Kunimine Co. with the cation-exchange capacity of $108.6 \mathrm{meq} / 100 \mathrm{~g}$. The organophilic clay was prepared by cation exchange reactions of natural clay (aqueous dispersion) with $20 \%$ aqueous solution of dimethyl methylphenyl octadecyl (DMPO), dimethyl dioctadecyl (DMDO), trimethyl octadecyl (TMO), and methyl hydroxy ethyl octadecyl (MHEO) ammonium chloride (Fig. 1). Procedures of these organo-modifications of montorillonites are described in reference. ${ }^{8}$ 2.2 Formation of organized molecular films

The organo-modified clays were spread from
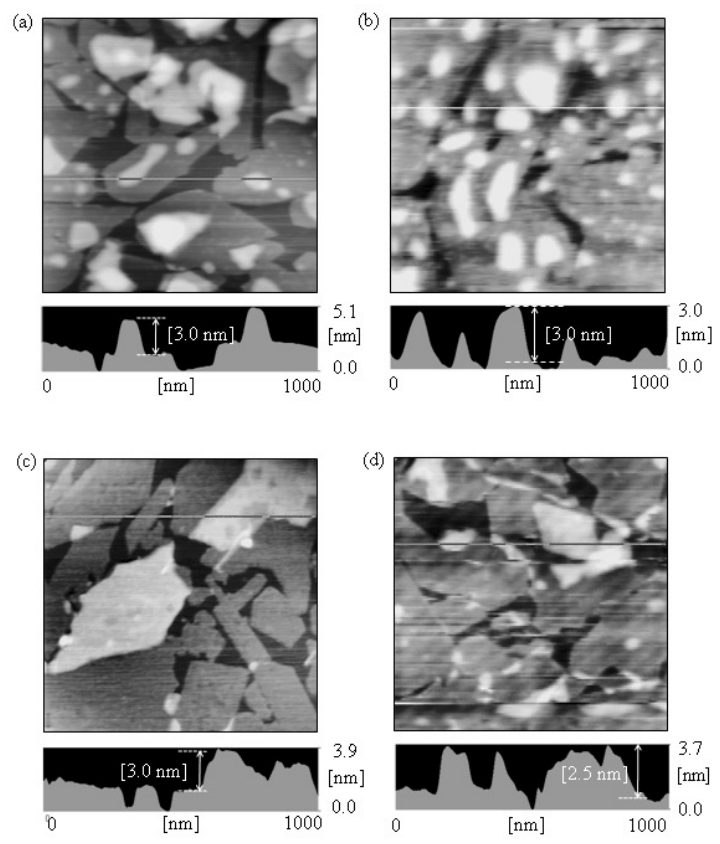

Figure 3 AFM images with the height information of Z-type monolayers of organo-modified clays; (a) DMPOMMT, (b) DMDO-MMT, (c) MHEOMMT, and (d) TMO-MMT (transferred at $15 \mathrm{mNm}^{-1}, 1 \times 1 \mu \mathrm{m}^{2}$ scales $)$.
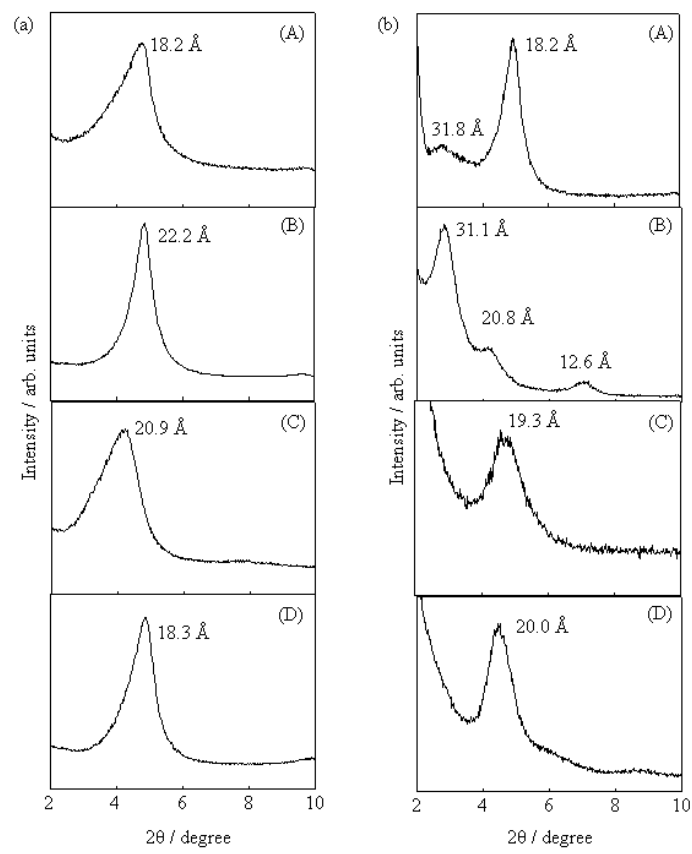

Figure 4 (a) X-ray powder diffraction profiles, and (b) Out-of plane XRD profiles of LB films (20 layers, $\left.15 \mathrm{mN} / \mathrm{m}, 15{ }^{\circ} \mathrm{C}\right)$ for organo-modified clays: (A) DMPO-MMT, (B) DMDO-MMT, (C) MHEO-MMT, (D) TMO-MMT.

toluene solution (approximately $10^{-4} \mathrm{M}$ ) onto distilled water (approximately $18 \mathrm{M} \Omega \cdot \mathrm{cm}$ ), respectively. The $\pi-\mathrm{A}$ isotherms were measured by a FACE film balance (Kyowa Kaimen Co.) at $15{ }^{\circ} \mathrm{C}$. These monolayers were transferred onto 
(a)

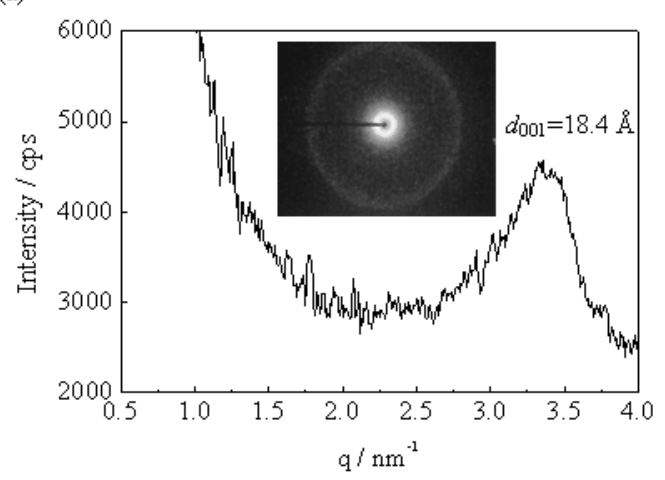

(b)

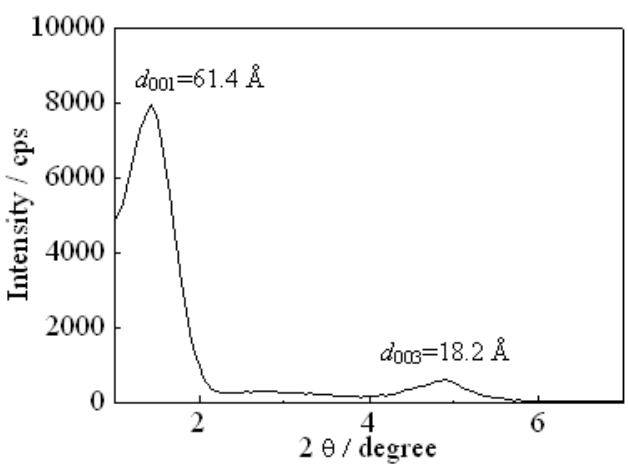

Figure 5 (a) SAXS pattern and profile of DMPO-MMT in bulk and (b) Out-of plane XRD profile of LB film for DMPO-MMT.

glass (XRD samples) or mica (AFM samples) substrates at $15^{\circ} \mathrm{C}$ using the LB method.

2.3. Wide-angle X-ray diffraction (WAXD) of bulk powder and out-of plane X-ray diffraction of organized molecular films

The structures of the organo-modified MMTs in bulk and the layer structures of organized molecular films were characterized with a RAD-rA diffractometer (RIGAKU Co.; in the case of organized molecular films, the out-of plane X-ray diffraction method was used). $\mathrm{Ni}$-filtered $\mathrm{Cu} K \alpha$ radiation (wavelength $\lambda=0.154$ $\mathrm{nm}$ ) was generated at $40 \mathrm{kV}$ and $100 \mathrm{~mA}$. The diffracted X-ray beam was monochromatized by a pyrographite monochromatic system and monitored by a scintillation counter.

2.4. Small-angle $X$-ray scattering (SAXS)

The crystalline morphology of the organo-modified clays was characterized with a SAXS instrument (M18XHF, MAC Science Co.) comprised of an $18 \mathrm{~kW}$ rotating-anode $\mathrm{X}$-ray generator with a $\mathrm{Cu}$ target $(\lambda=0.154 \mathrm{~nm})$ operated at $50 \mathrm{kV}$ and $300 \mathrm{~mA}$. This instrument was equipped with a pyrographite monochromator, pinhole collimation system $(\phi \sim 0.3,0.3,1.1 \mathrm{~mm})$, vacuum chamber for the scattered beam path, and two-dimensional imaging plate detector (DIP-220).

\subsection{Atomic force microscopy (AFM)}

The surface morphologies of the transferred monolayers were observed using a scanning probe
Table 1 Values of long spacings of organo-modified clays (a) in bulk and (b) in the LB films.

(a)

\begin{tabular}{|l|c|c|c|}
\hline & $2 \theta$ (degree) & $d$-Spacing $(\AA)$ & $h k l$ \\
\hline DMPO-MMT & 4.84 & 18.3 & 001 \\
\hline DMDO-MMT & 3.98 & 22.2 & 001 \\
\hline MHEO-MMT & 4.22 & 20.9 & 001 \\
\hline TMO-MMT & 4.84 & 18.3 & 001 \\
\hline
\end{tabular}

(b)

\begin{tabular}{|l|c|c|c|}
\hline & $2 \theta$ (degree) & $d$-Spacing $(\AA)$ & $h k l$ \\
\hline \multirow{3}{*}{ DMPO-MMT } & 1.42 & 61.4 & 001 \\
\cline { 2 - 4 } & 2.78 & 31.8 & 002 \\
\cline { 2 - 4 } & 4.90 & 18.0 & 003 \\
\hline \multirow{3}{*}{ DMDO-MMT } & 1.44 & 62.2 & 001 \\
\cline { 2 - 4 } & 2.78 & 31.1 & 002 \\
\cline { 2 - 4 } & 4.90 & 18.0 & 003 \\
\cline { 2 - 4 } & 7.02 & 12.6 & 005 \\
\hline \multirow{2}{*}{ MHEO-MMT } & 1.53 & 57.9 & 001 \\
\cline { 2 - 4 } & 4.62 & 19.1 & 003 \\
\hline \multirow{2}{*}{ TMO-MMT } & 1.47 & 60.0 & 001 \\
\cline { 2 - 4 } & 4.40 & 20.0 & 003 \\
\hline
\end{tabular}

microscope (Seiko Instrument, SPA300 with SPI-3800 probe station) using microfabricated rectangular $\mathrm{Si}$ cantilevers with integrated pyramidal tips with a constant force of $1.3 \mathrm{Nm}^{-1}$. 2.6 In-plane X-ray diffraction

The in-plane spacing of the two-dimensional lattice of the films was determined by analysis using an X-ray diffractometer for twodimensional geometrical arrangements (Bruker AXS, MXP-BX, $\mathrm{CuK \alpha}$ radiation, $40 \mathrm{kV}, 40 \mathrm{~mA}$, an instrument specially made to order) equipped with a parabolic graded multilayer mirror.

\section{RESULTS AND DISCUSSION}

Figure 2 shows the $\pi-\mathrm{A}$ isotherms of monolayer on the water surface of organo-modified MMTs. From these isotherms, we recognize formation of extremely stable monolayers. In this measurement, we represent two-type expression as "compression percentage vs. surface pressure" and "mean area per repeating units vs. surface pressure" plots because surface modification ratios are not always constant each other and $100 \%$ coverage.

Figure 3 shows the AFM images of Z-type monolayer on solid transferred by LB method of organo-modified MMTs. From this measurement, it is found that surface morphology of these monolayers of organo-modified clays indicates heterogeneous modification ratio on montmorillonite surface.

Figure 4 shows comparison between wide-angle X-ray diffraction profiles in bulk and LB films of organo-modified MMTs. In the case of LB film, this measurement corresponds to out-of plane XRD along the layer axis of molecules (c-axis). In the bulk state (Fig. 4(a)), all four organo-modified MMTs indicate single diffraction peak around $20 \AA$ on these profiles. Based on the results of SAXS measurement in bulk (Fig. 5(a)), it is found that all WAXD peaks 


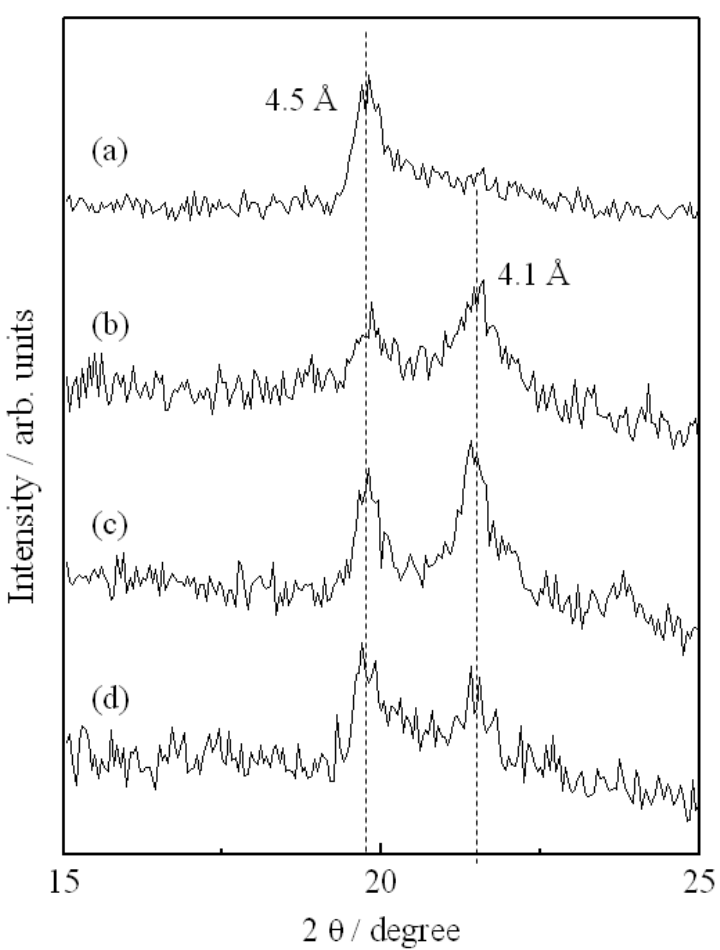

Figure 6 In-plane XRD profiles of LB films (20 layers, $15 \mathrm{mNm}^{-1}, 15^{\circ} \mathrm{C}$ ) for organo-modified montorillonites; (a) DMPO-MMT, (b) DMDO-MMT, (c) MHEO-MMT and (d) TMO-MMT.

around $20 \AA$ correspond to first order diffraction peaks along the $c$-axis $(001)$.

From the results of out-of plane XRD at low angle region (Fig. 5(b)) to the LB film, it is found that organo-modified MMTs indicate long spacings around $60 \AA$. Therefore, diffraction peaks confirmed at wide-angle region (Fig. 4(b)) correspond to higher order diffraction. These results are summarized in table 1. Comparison between regularity of layer structure in bulk and their LB films, organo-modified MMTs form the extremely developed layered organization in their LB multilayers.

Figure 6 shows in-plane XRD profiles of LB films of organo-modified MMTs. In order to estimate the packing mode of long alkyl chain in the organo-modified part, this measurement are carried out at the region related to sub-cell formation of hydrocarbons $\left(2 \theta=10 \sim 30^{\circ}\right)$. The organo-clay of only DMPO-MMT indicates single peak at $4.5 \AA$ and other organo-modified clays show double peaks at 4.1 and $4.5 \AA$ in the profiles of LB mulitlayers. Figure 7 shows schematic illustration of packing models for hydrocarbons in organo-modified parts on montorillonite in the LB films. In the two-dimensional plane, alkyl-chains of DMPO-MMT packed hexagonally in their LB film. On the other hand, hydrocarbons of DMDO-MMT, MHEO-MMT, and TMO-MMT commonly form the two-dimensional orthorhombic lattice in the film plane. This structural change may show the influence on the existence of bulky phenyl groups in DMPO regent
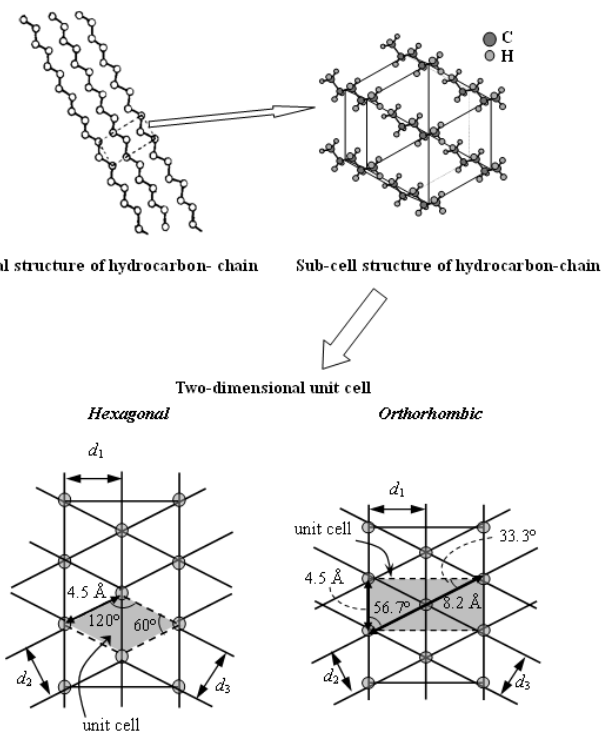

$(a=b \neq c$

$\alpha=\beta=90^{\circ}, \gamma=120^{\circ}$

$d_{1}=d_{2}=d_{2}=4.5 \AA$

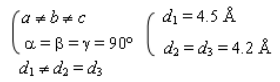

Figure 7 Two-dimensional lattice formed by long alkyl chain of organo-modified montorillonites in their LB films.

and its steric hindrance.

These experimental findings show the formation of highly order layered organization of organo-modified MMTs constructed by LB method. In this case, hydrocarbons in organo-modified part are closely packed as two-dimensional lattice although organo-modified ratio on montorillonite does not always indicate higher values.

\section{REFERENCES}

[1] G. H. Beall, L. L. Hench, S. W. Freeman (Eds.), "Advances in Nucleation and Crystallization in Glasses", American Ceramics Society, pp. 5 (1971).

[2] T. J. Pimavaia, J.-R. Butruille, G. Alberti, T. Bein (Eds.), "Comprehensive Supramolecular Chemistry", vol. 7, Elsevier, Oxford, (1996). Chapter 7.

[3] A. J. Bard, T. Mallouk, R. W. Murray (Ed.), "Techniques of Chemistry Series", vol. 22, Cbapter 6, Wiley, New York, (1992).

[4] G. L. Gaines Jr, "Insoluble Monolayers at Liquid-Gas Interfaces", Wiley-Interscience, New York, (1966).

[5] Y. Hotta, M. Taniguchi, K. Innkai, A, Yamagishi, Langnuir, 12, 5195 (1996).

[6] Y. Umemura, A. Yamagishi, R. Schoonheydt, A. Persoons, F. De Schryver, Thin Solid Films, 388, 5 (2001).

[7] B. K. G. Theng, "The Chemistry of Clay-Organic Reactions", Adam Hilger, London, (1974).

[8] P. H. Nam, M. Kaneko, N. Ninomiya, A. Fujimori and T. Masuko, Polymer, 46, 7403 (2005).

(Received December 29, 2009; Accepted April 5, 2010) 